\title{
Compreendendo o Impacto de um Programa de Perda de Peso: os papéis do alcance, retenção e efetividade
}

\author{
Understanding the Impact of a Weight Loss Program: the roles of reach, \\ retention, and effectiveness
}

\author{
Comprendiendo el Impacto de un Programa de Pérdida de Peso: \\ los papeles del alcance, retención y efectividad
}

Recebido:15/07/2013

Aprovado: 23/02/2014

\author{
Paul Andrew Estabrooks ${ }^{1}$ \\ Mita Shah Bhagat ${ }^{2}$ \\ Fabio Araújo Almeida ${ }^{3}$ \\ Brenda Marie Davy ${ }^{4}$ \\ Wendy You ${ }^{5}$
}

O objetivo deste artigo é demonstrar os potenciais problemas na confiança das análises usando intenção de tratamento (ITT) quando o alcance inicial não é contabilizado. Dados de arquivo de sete locais de trabalho ( $\mathrm{n}=10.513$ funcionários) que completaram um programa de perda de peso foram utilizados para determinar: (a) a eficácia do programa no final de três a seis meses de participação; (b) o alcance do programa na população de funcionários elegíveis; e (c) a proporção da população alvo de funcionários que, em última análise, beneficiaram-se do programa e o grau desses benefícios (como a efetividade que considera o alcance e retenção). Análises de ITT utilizando o valor de peso inicial para participantes perdidos a desistência revelou que os participantes perderam uma significativa $(\mathrm{p}<0,01)$, porém modesta, quantidade de peso em três meses (0,95 quilos) e em seis meses (1,09 quilos) de participação. A seguir foram realizadas análises para determinar a proporção total da força de trabalho que se beneficiou (peso perdido) em 6 meses. Dos 1.607 participantes que foram mantidos por pelo menos 6 meses, 1088 foram bem sucedidos na perda de peso apresentando uma perda média de 4,3 $\mathrm{kg}$ (95\% CI: 4,0 a 4,5Kg), indicando uma perda clinicamente significativa de $4,4 \%$ do peso corporal inicial. Assim, 10,1\% da população total de funcionários se beneficiaram com o programa de perda de peso e perderam uma quantidade clinicamente relevante de peso. Os resultados deste estudo indicam que a presença de dados de alcance combinados com dados de efetividade podem auxiliar empregadores na tomada de decisões mais sofisticadas ao escolher um programa de perda de peso comercial para os seus funcionários, quando comparado à análise tradicional de ITT.

Descritores: Promoção da saúde; Programas de redução de peso; Avaliação de programas e projetos de saúde; Obesidade; Efetividade.

\footnotetext{
${ }^{1}$ Educador Físico. Mestre em Cinesiologia. Doutor em Cinesiologia. Professor dos Cursos de Mestrado e Doutorado em Nutrição Humana, Alimentação e Exercício. Universidade Virginia Tech., EUA estabrkp@vt.edu

${ }^{2}$ Nutricionista. Mestre em Ciência dos Alimentos, Nutrição e Dietética. Mestre em Ciência da Nutrição Humana, Alimentação e Exercício. Membro do Conselho de Promoção da Saúde de Massachusetts. Pesquisadora do Departamento de Nutrição Humana, Alimentos e Exercício. Universidade Virginia Tech., EUA.

${ }^{3}$ Assistente Social. Especialista em Práticas Comunitárias. Mestre em Serviço Social com ênfase em Prática Comunitária e Desenvolvimento Internacional. Doutor em Serviço Social. Professor dos Cursos de Mestrado e Doutorado em Nutrição Humana, Alimentação e Exercício. Universidade Virginia Tech., EUA.

${ }^{4}$ Nutricionista. Mestre em Fisiologia do Exercício. Doutora em Nutrição Humana. Professora dos Cursos de Mestrado e Doutorado em Nutrição Humana, Alimentação e Exercício. Universidade Virginia Tech, EUA.

${ }^{5}$ Bacharel em Artes. Especialista em Comércio Internacional. Doutora em Economia Agrária. Departamento de Agricultura Aplicada. Professora do Mestrado em Agricultura e Economia Aplicada e do Doutorado em Economia. Universidade Virginia Tech., EUA.
} 
The objective of this paper is to demonstrate potential problems of relying on intention to treat analyses when initial reach is not accounted for. Archival data from seven worksites ( $n=10,513$ employees) that completed a weight loss program was used to determine (a) the effectiveness of the program at the end of three and six months of participation, (b) the reach of the program into the eligible employee population, and (c) the proportion of the targeted employee population that ultimately benefited from the program and the degree to which they benefited (i.e., effectiveness that accounts for reach and retention). Intention to treat analyses using baseline value carried forward for participants lost to attrition revealed that participants lost a significant ( $\mathrm{p}<.01$ ), yet modest, $2.1 \mathrm{lbs}$ of weight at 3 and $2.4 \mathrm{lbs}$ of weight at 6 months of participation. Follow-up analyses were conducted to determine the overall proportion of the workforce that benefited (i.e., lost weight) at 6 months. Of the 1607 participants who were retained at 6 months, 1088 were successful in losing weight and lost, on average 9.4 pounds ( $95 \%$ CI: 8.8 to 9.9 pounds), a clinically significant $4.4 \%$ of initial body weight. Thus, $10.1 \%$ of the total employee population benefited from the weight loss program and lost a clinically relevant amount of weight. The findings of this study indicate that presenting reach by effectiveness data to the employers could help them in making more sophisticated decisions while choosing a commercial weight loss program for their employees when compared to traditional intention to treat analyses.

Descriptors: Health promotion; Weight reduction programs; Program evaluation; Obesity; Effectiveness.

El objetivo de éste artículo es demostrar los potenciales problemas en la confianza de los análisis usando la intención de tratamiento (ITT) cuando el alcance inicial no es contabilizado. Datos de archivo de siete locales de trabajo ( $n=10.513$ funcionarios) que completaron un programa de pérdida de peso fueron utilizados para determinar: (a) la eficacia del programa al final de tres a seis meses de participación; (b) el alcance del programa en la población de funcionarios elegibles; y (c) la proporción de la población albo de funcionarios que en último análisis, se beneficiaron del programa y el grado de esos beneficios (como la eficacia que considera el alcance y retención). Análisis de ITT utilizando el valor de peso inicial para participantes perdidos en la desistencia reveló que los participantes perdieron una significativa ( $p>0,01)$, aunque modesta, cantidad de peso en tres meses $(0,95$ Kilos) y en seis meses (1,09 Kilos) de participación. Luego fueron realizados análisis para determinar la proporción total de la fuerza de trabajo que se benefició (peso perdido) en 6 meses. De los 1607 participantes que fueron mantenidos por lo menos por 6 meses, 1088 tuvieron éxito en la pérdida de peso presentando una pérdida media de 4,3 $\mathrm{kg}$ (95\% CI: 4,0 a 4,5kg), indicando una pérdida clínicamente significativa de 4,4\% del peso corporal inicial. Así, 10,1\% de la población total de funcionarios se beneficiaron con el programa de pérdida de peso y perdieron una cantidad clínicamente relevante de peso. Los resultados de éste estudio indican que la presencia de datos de alcance combinados con datos de eficacia pueden auxiliar empleadores en la toma de decisiones más sofisticadas al elegir un programa de pérdida de peso comercial para sus funcionarios, comparado al análisis tradicional de ITT.

Descriptores: Promoción de la salud; Programas de reducción de peso; Evaluación de programas y proyectos de salud; Obesidad; Efetividad. 


\section{INTRODUÇÃO}

$\mathrm{H}$ á evidências claras de que a prevalência de sobrepeso e obesidade refletem uma epidemia internacional e que leva a problemas graves que influenciam na saúde, nos custos pessoais e sociais, assim como na produtividade da força de trabalho ${ }^{1}$.

Para uma intervenção de gerenciamento de peso com base no local de trabalho ter um forte impacto deve-se atingir uma parcela ampla da população de funcionários com sobrepeso e obesidade ${ }^{2}$.

Diversos investigadores tentaram melhorar o alcance dos programas, oferecendo intervenções que podem ser aplicadas por via eletrônica. Embora o pressuposto seja de que os programas via internet terão maior alcance (em parte devido à remoção da barreira do tempo tomado por reuniões em pequenos grupos) há uma escassez de estudos que demonstram número de funcionários que são atingidos por programas baseados na internet.

Numa avaliação de seis estudos que utilizaram intervenções de promoção da saúde baseado nos locais de trabalho pela internet, apenas três desses estudos relataram alcance da intervenção que variaram de $6,0 \%$ a $60,0 \%$ da população $0^{3-8}$. Além disso, o alcance é definido como uma variável temporal fixa (alcance/reach - o número, proporção e representatividade dos participantes quando eles aderiram ao programa). Esta definição pode ser um pouco limitada na medida em que aqueles alcançados no início de um programa não são geralmente as mesmas pessoas que são mantidas durante o programa.

Uma série de estudos realizados anteriormente reporta efetividade em termos de número médio de quilos perdidos pelos participantes. E para contabilizar baixa retenção, análises de Intenção de Tratamento (ITT) são consideradas necessárias a fim de manter a validade interna ${ }^{9}$. Análise de ITT também são utilizadas regularmente para determinar a efefividade porque incluem todos os participantes que tenham iniciado o programa e imputam dados de seguimento para contabilizar a perda de participantes por abandono durante o curso de um programa ${ }^{10}$.

Pela perspectiva do RE-AIM, informações acerca do alcance e efetividade são necessárias ${ }^{11}$. Para combinar alcance e efetividade, os investigadores poderiam simplesmente relatar o número e a proporção de sobrepeso e obesidade na população de funcionários envolvidos no programa e, em seguida, a magnitude da perda de peso resultante, geralmente, a partir de análises de ITT.

No entanto, isso poderia mascarar resultados significativos para uma pequena parte da população do local de trabalho. Por exemplo, utilizando resultados de uma revisão sistemática de pesquisas sobre perda de peso no local de trabalho o empregador poderia entender que a perda de peso médio por empregado participante é cerca de 1,6 quilos aos seis meses (utilizando a metodologia ITT) e que aproximadamente $20 \%$ dos funcionários participariam ${ }^{12}$. Esta informação dificilmente seria atraente para um coordenador de saúde e bem-estar do local de trabalho interessado em implementar um programa de perda de peso.

Neste trabalho, propõe-se o uso de técnicas analíticas de dados que representam as dimensões alcance (Reach) e Efetividade do modelo RE-AIM ${ }^{11,13}$, bem como a retenção por mais de três a seis meses. Com tal finalidade, utilizou-se o banco de dados de um programa de perda de peso disponível comercialmente via internet com base no local de trabalho. 0 objetivo deste estudo foi determinar o alcance deste programa para os funcionários nos locais de trabalho e examinar a efetividade no contexto do alcance e da retenção (a proporção da população alvo de funcionários que, em última análise, beneficiaram-se do programa e o grau deste beneficio). 


\section{MÉTODO}

Este é um estudo de arquivos de banco de dados secundários de locais de trabalho de um programa de perda de peso disponível comercialmente via internet com base no local de trabalho (Programa IncentaHEALTH ${ }^{T M}$ ) implementado em sete locais de trabalho.

O banco de dados incluiu informação sobre idade e sexo de participantes coletada no início do programa, a retenção (três a seis meses) e a perda de peso (após três a seis meses de participação no programa). Os sete locais de trabalho utilizados representam o número total que tinha contratado o programa comercial e que tinham o arquivo de dados com todas as características necessárias disponíveis na época do estudo.

Todos os funcionários que iniciaram o programa foram incluídos nas análises. Todos os dados dos participantes do programa foram codificados e tiveram total anonimato em relação à equipe de pesquisa. Os critérios de elegibilidade do programa incluíram participantes que fossem funcionários dos locais de trabalho e possuissem IMC maior ou igual a 25 .

0 programa IncentaHEALTH ${ }^{T M}$ incluia e-mail de apoio diário, acesso a um site repleto de informações estratégicas e educacionais, bem como incentivos financeiros mensais modestos.

Os incentivos financeiros foram baseados na percentagem de peso corporal perdido (cinco dólares/mês por 5\% de peso perdido), registrados em avaliações trimestrais. Os participantes foram incentivados a adequar suas dietas de acordo com suas preferências, assim como escolher onde realizar o programa de exercícios (em casa ou na academia) e em qual nível de exercício começariam o programa (iniciante, intermediário ou avançado). 0 empregador cobriu o custo do programa.

0 peso de cada participante foi avaliado usando uma balança calibrada e validada em cada local de trabalho no princípio do programa e depois a cada três meses. A balança calibrada continha uma câmera digital embutida para capturar a foto dos participantes durante a pesagem para fornecer uma verificação de identidade e para fins de motivação (participantes podiam acompanhar o seu progresso ao longo do tempo). Informações sobre o número total de empregados foram fornecidas por todos os locais de trabalho.

Para o presente estudo, a indisponibilidade de dados sobre o peso em três a seis meses foi usada para categorizar os participantes como abandonos.

Para determinar o alcance do programa em relação à população de funcionários elegíveis, foram realizados cálculos de frequência simples utilizando como denominador a população total de funcionários e a população total projetada como elegível (ou seja, 67,0\% da população adulta dos EUA classificada com sobrepeso ou obesa) e como numerador os funcionários participantes.

Para determinar a efetividade do programa, ao final de três a seis meses de participação, uma análise de ITT foi realizada utilizando testes t pareados usando o valor de peso inicial para participantes que abandonaram com os valores aos três a seis meses. Para determinar a proporção da população alvo de funcionários que, em última análise, beneficiaram-se do programa e o grau desse beneficío, a avaliação do peso dos participantes aos seis meses foram analisadas com base em todos os que perderam peso e os que permaneceram por seis meses.

Por sua vez, o cálculo da proporção da população que se beneficiou, usou-se como denominadores a população total do local de trabalho e a população elegível total. Como numeradores foram utilizadas a proporção de quem se beneficiou e o número de funcionários que perderam peso.

\section{RESULTADOS}

Um total de 10.513 pessoas eram funcionários dos locais de trabalho (Figura 1). Destes, 4.233 funcionários eram elegíveis (IMC $>=25$ ) e aderiram ao programa. 0 alcance (Reach) do programa em relação à 
população total de funcionários foi de foi de aproximadamente 60,0\%. Dos aproximadamente $40,0 \%$. No entanto, participantes $66,0 \%$ eram mulheres e a quando se considerou os funcionários que idade média foi 44 anos. seriam elegíveis para o programa, o alcance

Figura 1. Diagrama do fluxo de recrutamento de participantes e retenção ao longo de 6 meses de implementação do programa. Estado do Colorado, EUA, 2010.

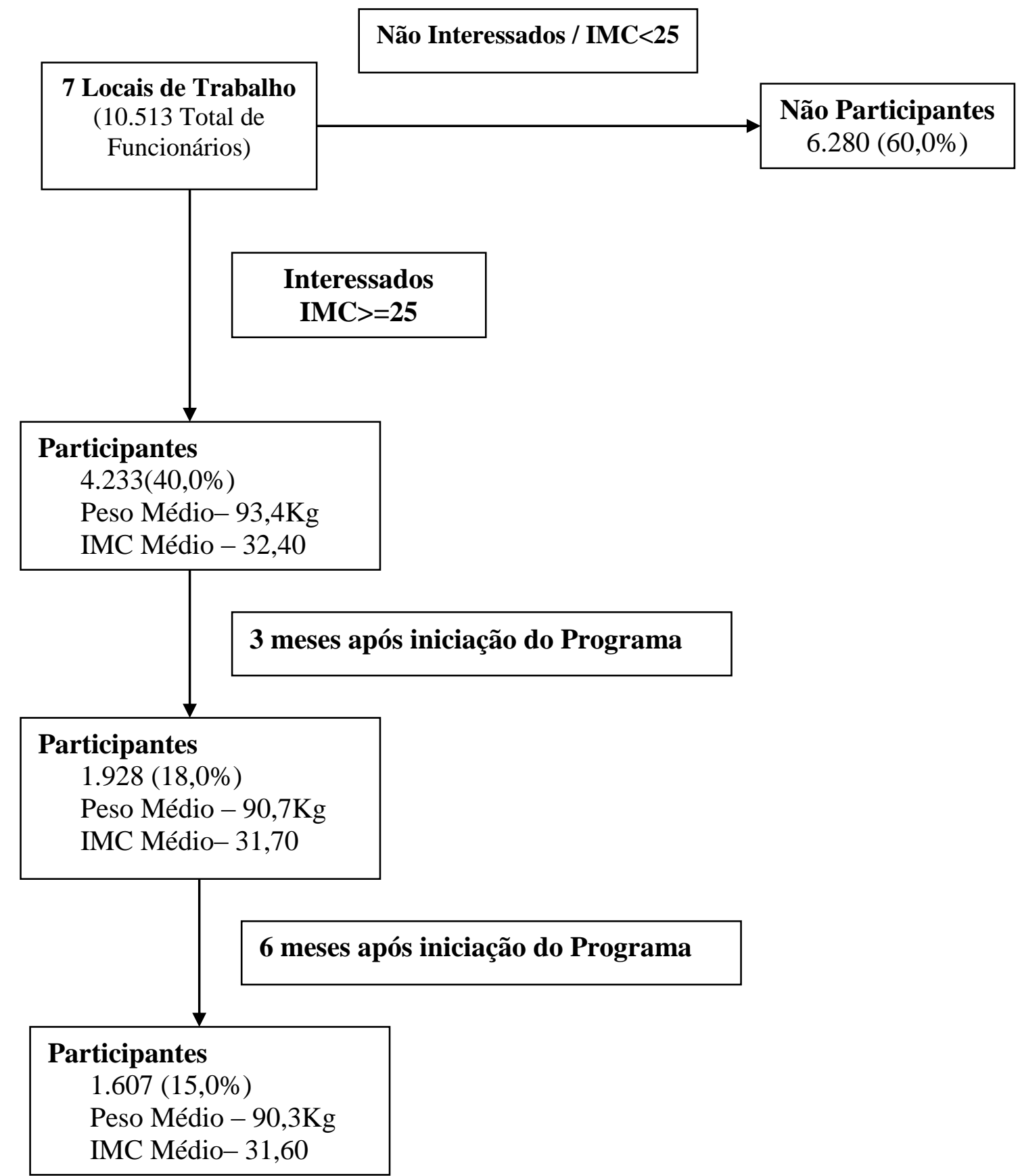

0 peso médio dos participantes foi de $93,44( \pm 19,50) \mathrm{Kg}$ e o IMC inicial foi de 32,4 . Aos três meses, os participantes perderam uma quantidade significativa de peso $(\mathrm{t}$ $=21,75, \mathrm{p}<0,001)$, em média $0,95( \pm 2,86) \mathrm{kg}$, com um intervalo de confiança de 95,0\% de 0,86 a 1,04 Kg. Similarmente, aos seis meses os participantes perderam uma quantidade significativa de peso $(t=20,43, p<0,001)$, em média $1,09( \pm 3,58) \mathrm{kg}$, com intervalo de confiança de $95,0 \%$ de 1,0 a 1,22 Kg. Esta perda de peso, com base em análise de ITT, representou aproximadamente $1,75 \%$ do peso corporal inicial perdido, em média, por 
cada participante.

Análises de seguimento foram conduzidas para determinar a proporção total da força de trabalho, que se beneficiou (peso perdido) em seis meses. Três meses após o início, 1.928 participantes $(28,0 \%$ da população elegível e $18,0 \%$ da população total) permaneceram, e aos seis meses 1.607 participantes $(23,0 \%$ da população elegível e $15,0 \%$ da população total) permaneceram no programa. Dos 1.607 participantes que foram mantidos em seis meses, 1.088 tiveram sucesso na perda de peso e perderam em média 4,26 Kg (95,0\% CI: 3,99-4,49 kg), uma perda clinicamente significativa de - 4,4\% do peso corporal inicial. Desta forma, 10,1\% da população total e $16,0 \%$ da população elegível de funcionários se beneficiaram desse programa de perda de peso, de forma clinicamente significativa.

\section{DISCUSSÃO}

Alguns dos estudos anteriores relatam uma taxa muito superior de efetividade ${ }^{14-16}$; entretanto, esses estudos geralmente envolvem intervenções de alta intensidade e sua análise final é baseada em amostras homogêneas de indivíduos altamente motivados que estão disponíveis para avaliação final.

Esses estudos fornecem informações com forte validade interna; mas do ponto de vista de validade externa, é improvável que as amostras sejam representativas dos participantes e localidades. Por outro lado, raramente estudos com intervenções de maior intensidade apresentam dados sobre 0 alcance na população alvo; ainda mais: pode ser que haja uma relação inversa entre o alcance e a retenção.

Análises de ITT são consideradas meio cientificamente aceitável de se relatar dados sobre programas de perda de peso. Quando os dados estudados foram analisados utilizando esse método, foram encontradas mudanças estatisticamente significativas, mas modesta, no peso, na melhor das hipóteses. Apesar disso, quando os dados de alcance, efetividade e retenção são compilados em conjunto, considerando que $10,0 \%$ da população do local de trabalho em geral se benefíciou em um nível clínicamente significativo, verificou-se que o programa foi atraente para os empregadores.

$\mathrm{Na}$ verdade, programas típicos de gestão de doenças no local de trabalho são direcionados a doenças com taxa de prevalência menores que $10,0 \%$, o que sugerem que esse tipo de programa de perda de peso no local de trabalho possa ter um impacto maior do que a maioria das abordagens de gerenciamento de doenças. É evidente que métodos diferentes de apresentação dos mesmos dados podem levar a conclusões muito diferentes acerca de um programa.

A utilização de dados de alcance, retenção e efetividade fornecem uma rica fonte de informações que verdadeiramente indicam a proporção de uma população de funcionários que poderiam ser beneficiados e em que intensidade.

Tem-se de reconhecer algumas limitações da abordagem deste estudo. Primeiro, que este foi um estudo piloto em que foram utilizados dados secundários, e não foi realizado grupo controle. Assim sendo, este estudo possui uma capacidade limitada de aferir mudanças individuais e se o programa levou a essas mudanças de comportamento. Do mesmo modo, como foram utilizados dados de arquivo de um programa comercial para perda de peso não houve informações adicionais sobre a demografia dos participantes para determinar a representatividade.

\section{CONCLUSÃO}

0 principal objetivo deste trabalho foi destacar as diferentes formas que dados acerca do alcance (Reach), retenção e efetividade poderiam ser relatados. Análises de ITT com manutenção de imputação de valores do princípio do programa indicaram uma perda significativa, porém pequena, de peso.

Os profissionais de saúde dos locais de trabalho são confrontados com inúmeras decisões relacionadas a 
programas de promoção da saúde que serão oferecidos para os seus trabalhadores. Entretanto, a maioria dos estudos não documenta ou controla as taxas de abandono; sendo assim, os dados de efetividade refletem apenas os melhores cenários de cada caso.

0 método aqui descrito pode fornecer um modelo para programas comerciais e de pesquisa documentarem a proporção dos trabalhadores que, em última análise, beneficiam-se de um programa de bem-estar e do grau em que esse benefício é clinicamente importante.

\section{REFERÊNCIAS}

1. Whitmer RW, Pelletier KR, Anderson DR, Baase CM, Frost GJ. A wake-up call for corporate America. J Occup Environ Med. 2003; 45(9):91625.

2. Estabrooks PA, Glasgow RE. Worksite interventions. In: Ayers $\mathrm{S}$ et al, editores. Cambridge handbook of psychology, health and medicine. New York: Cambridge University press; 2007. p. 407-13. 3. Aldana S, Barlow M, Smith R, Yanowitz F, Adams T, Loveday L, et al. A worksite diabetes prevention program: two-year impact on employee health. AAOHN J. 2006; 54(9):389-95.

4. Faghri PD, Omokaro C, Parker C, Nichols E, Gustavesen S, Blozie E. E-technology and pedometer walking program to increase physical activity at work. J Prim Prev. 2008; 29(1):73-91.

5. Tate DF, Jackvony EH, Wing RR. Effects of Internet behavioral counseling on weight loss in adults at risk for type 2 diabetes: a randomized trial. JAMA. 2003; 289(14):1833-6.

6. Petersen R, Sill S, Lu C, Young J, Edington DW. Effectiveness of employee internet-based weight management program. J Occup Environ Med. 2008; 50(2):163-71

7. Prochaska JO, Butterworth S, Redding CA, Burden V, Perrin N, Leo M, et al. Initial efficacy of MI, TTM tailoring and HRI's with multiple behaviors for employee health promotion. Prev Med. 2008; 46(3):226-31.

8. White K, Jacques PH. Combined diet and exercise intervention in the workplace: effect on cardiovascular disease risk factors. AAOHN J. 2007; 55(3):109-14.

9. Shadish WR, Cook TD, Campbell DT. Experimental \& quasi-experimental designs for general causal inference. New York: Houghton Mifflin Company; 2001.

10. Atlantis E, Chow CM, Kirby A, Fiatarone Singh MA. Worksite intervention effects on physical health: a randomized controlled trial. Health Promot
Int. 2006; 21(3):191-200.

11. Glasgow RE, Magid DJ, Beck A, Ritzwoller D, Estabrooks PA. Practical clinical trials for translating research to practice: design and measurement recommendations. Med Care. 2005; 43(6):551-7.

12. Anderson LM, Quinn TA, Glanz K, Ramirez G, Kahwati LC, Johnson DB, et al. The effectiveness of worksite nutrition and physical activity interventions for controlling employee overweight and obesity: a systematic review. Am J Prev Med. 2009; 37(4):340-57.

13. Almeida FA, Brito FA, Estabrooks PA. Modelo RE-AIM: tradução e adaptação cultural para o Brasil. REFACS. 2013; 1(1):6-16.

14. Jeffery RW, Forster JL, Snell MK. Promoting weight control at the worksite: a pilot program of self-motivation using payroll-based incentives. Prev Med. 1985; 14(2):187-94.

15. Lloyd KB, Krueger KP, Moore RT, Walters NB, Eichner SF, Fanning K. Impact of a workplace health and wellness pharmaceutical care service on the weight and obesity classification of employees. $\mathrm{J}$ Am Pharm Assoc. 2002; 42(1):118-20.

16. Prochaska JO, Norcross JC, Fowler JL, Follick MJ, Abrams DB. Attendance and outcome in a work site weight control program: processes and stages of change as process and predictor variables. Addict Behav. 1992; 17(1):35-45.

\section{CONTRIBUIÇÕES}

Paul Andrew Estabrooks foi responsável pela condução do estudo apresentado, contribuiu no desenho, na análise de dados, na interpretação e discussão dos resultados, e redação do artigo;

Mita Shah Bhagat contribuiu no desenho, na coleta de dados, e redação do artigo;

Fabio Araújo Almeida contribuiu no desenho, na interpretação e discussão dos resultados, e redação do artigo;

Brenda Marie Davy contribuiu no desenho, na interpretação e discussão dos resultados, redação do artigo;

Wendy You conduziu a análise de dados, contribuiu na interpretação e discussão dos resultados, e redação do artigo.

\section{AGRADECIMENTOS}

Este estudo foi apoiado pelo National Institute on Diabetes and Digestive and Kidney Diseases (Grant R01DK 071664-04 and R01DK 07166404S1) (Estabrooks, PI). 0 conteúdo aqui apresentado é de única responsabilidade dos autores e não representa necessariamente opiniões oficiais do National Institute of Diabetes and Digestive and Kidney Diseases ou do National Institutes of Health. 